\title{
Effect of an in-plane magnetic field on the interlayer phase coherence in the extreme-2D organic superconductor $\kappa-(\mathrm{BEDT}-\mathrm{TTF})_{2} \mathrm{Cu}(\mathrm{NCS})_{2}$
}

\author{
A. E. Kovalev, ${ }^{1}$ S. Takahashi, ${ }^{1}$ S. Hill, ${ }^{1,}{ }^{*}$ and J. S. Qualls ${ }^{2}$ \\ ${ }^{1}$ Department of Physics, University of Florida, Gainesville, FL 32611,USA \\ ${ }^{2}$ Department of Physics, Wake Forest University, Winston-Salem, NC 27109, USA
}

(Dated: October 31, 2018)

\begin{abstract}
Using a high-sensitivity cavity perturbation technique (40 to $180 \mathrm{GHz}$ ), we have probed the angle dependent interlayer magneto-electrodynamic response within the vortex state of the extreme twodimensional organic superconductor $\kappa$-(BEDT-TTF $)_{2} \mathrm{Cu}(\mathrm{NCS})_{2}$. A previously reported Josephson plasma resonance [M. Mola et al., Phys. Rev. B 62, 5965 (2000)] exhibits a dramatic re-entrant behavior for fields very close $\left(<1^{\circ}\right)$ to alignment with the layers. In this same narrow angle range, a new resonant mode develops which appears to be associated with the non-equilibrium critical state. Fits to the angle dependence of the Josephson plasma resonance provide microscopic information concerning the superconducting phase correlation function within the vortex state. We also show that the effect of an in-plane magnetic field on the temperature dependence of the interlayer phase coherence is quite different from what we have previously observed for perpendicular magnetic fields.
\end{abstract}

Keywords: Josephson Plasma resonance; Organic superconductor; Vortices

Corresponding Author:

Dr. Stephen Hill

Department of Physics

University of Florida

Gainesville, FL 32611 (USA)

FAX: 1-352-392-3591

Email: hill@phys.ufl.edu 


\section{Introduction}

The quasi-two-dimensional (Q2D) organic superconductor $\kappa$-(BEDT-TTF $)_{2} \mathrm{Cu}(\mathrm{NCS})_{2}$ (BEDT-TTF denotes bis-ethylenedithio-tetrathiafulvalene, or ET for short) represents a model system for studying vortex physics in layered systems and, in particular, the influence of vortices on the interlayer phase coherence within the mixed state [1]. Like the high temperature superconductors (HTS), $\kappa$ - $(\mathrm{ET})_{2} \mathrm{Cu}(\mathrm{NCS})_{2}$ possesses a highly anisotropic layered structure with the superconducting ET planes separated by insulating anion layers [1]. The anisotropy parameter $\gamma$, defined as the ratio of the interlayer (currents $\| a$-axis) and in-plane (currents $\| b c$-plane) penetration depths $\left(\gamma \equiv \lambda_{a} / \lambda_{b c}\right)$, is thought to be in the range $50-200$ 2], i.e. similar to that of $\mathrm{Bi}_{2} \mathrm{Sr}_{2} \mathrm{CaCu}_{2} \mathrm{O}_{8+d}$ [3]. In contrast to the HTS, however, organic conductors are extremely clean, with very few crystal defects. Also, because of the reduced $\mathrm{T}_{c}(\sim 9-10 \mathrm{~K})$ and $\mathrm{B}_{c 2}(\sim 5 \mathrm{~T}$ for fields $\perp$ layers, and $30-35 \mathrm{~T}$ for fields || layers), one can probe much more of the temperature/field parameter space within the superconducting state than is currently possible in the HTS.

Our recent investigations of the microwave response of $\kappa$ - $(\mathrm{ET})_{2} \mathrm{Cu}(\mathrm{NCS})_{2}$ in the 30 to $200 \mathrm{GHz}$ range have shown that a Josephson Plasma Resonance (JPR) dominates the interlayer electrodynamics within the mixed state [4, 5]. The JPR frequency, $\omega_{p}$, depends explicitly on the Josephson coupling (interlayer phase coherence) between layers; indeed, $\omega_{p}$ is directly related to the spatial and temporal average of the function $\left\langle\cos \varphi_{n, n+1}(\mathbf{r})\right\rangle$, where $\varphi_{n, n+1}(\mathbf{r})$ is the gauge invariant difference in the phase of the order parameter between layers $n$ and $n+1$, at position $\mathbf{r}$ within the layers. Thus, the JPR serves as an extremely sensitive tool for probing the structure of vortex phases in this and other layered organic and inorganic superconductors [6]. Application of a magnetic field $\left(B_{z}\right)$ normal to the layers introduces pancake vortices into the sample, accompanied by large intra-layer phase fluctuations. Due to the weak nature of the Josephson coupling in the title compound, the pancake vortices in adjacent layers become decoupled at relatively weak applied fields $(\sim 10 \mathrm{mT})$. This decoupling transition dramatically suppresses the interlayer phase coherence $\left\langle\cos \varphi_{n, n+1}(\mathbf{r})\right\rangle$ which, in turn, suppresses $\omega_{p}$. Theoretical and experimental studies within the vortex liquid phase have shown that the field and temperature $(\mathrm{T})$ dependence of $\omega_{p}$ may be described as

$$
\omega_{p}^{2}\left(B_{z}, T\right) \propto B_{z}^{-\nu} T^{-1}
$$


with $\nu$ being slightly less than unity [4, [5, 6].

Until recently, our investigations were limited to the perpendicular field geometry. These studies revealed a transformation from a pinned vortex phase, to a depinned liquid state 4, 5]. Our improved experimental setup now allows angle dependent microwave impedance measurements of small single crystal samples (see below). Here, we report preliminary data mainly within the vortex liquid phase for fields close to alignment with the $b c$-plane (II highly conducting layers). Application of an in-plane field $\left(B_{x}\right)$ introduces an additional term in the phase difference $\varphi_{n, n+1}(\mathbf{r})$ due to the vector potential associated with $B_{x}$. This leads to a further suppression of $\omega_{p}$, which is directly related to the Fourier transform of the phase correlation function $S(\mathbf{r})=\left\langle\cos \varphi_{n, n+1}(\mathbf{r})-\cos \varphi_{n, n+1}(0)\right\rangle[\underline{6}$. Thus, angle dependent JPR measurements provide a direct means of extracting microscopic parameters associated with the superconducting state.

\section{Experimental}

Measurements were performed using a sensitive cavity perturbation technique described elsewhere [7]. As a source and detector, we use a millimeter-wave vector network analyzer (MVNA), enabling phase sensitive measurements covering the frequency range from 40 to $\sim 200 \mathrm{GHz}$. This phase sensitivity allows us to determine both components of the complex sample response [7], though we only plot the dissipative response in this article. A single crystal sample was placed within a cylindrical cavity such that a combination of in-plane and interlayer currents were excited; a detailed discussion of the electrodynamics is published elsewhere [8]. Interlayer currents penetrate deep $(100 \mu \mathrm{m}-1 \mathrm{~mm})$ into the sample due to the low conductivity in this direction. Dissipation then depends on the ratio of the interlayer and in-plane conductivities, as well as the relative dimensions of the sample. For this geometry, we have shown unambiguously that dissipation is dominated by the interlayer conductivity for the highly anisotropic organic conductors [7, 8].

A single $\kappa$ - $(\mathrm{ET})_{2} \mathrm{Cu}(\mathrm{NCS})_{2}$ crystal, with approximate dimensions $0.7 \times 0.5 \times 0.2 \mathrm{~mm}^{3}$, was used for these investigations. Temperature control in the 2 to $10 \mathrm{~K}$ range was maintained using a variable flow cryostat (Quantum Design PPMS), with Cernox resistance thermometers acting as temperature sensors. Smooth rotation of the entire rigid microwave probe, relative to the horizontal DC field produced by a $7 \mathrm{~T}$ split-pair magnet, was achieved via a room temperature stepper motor mounted at the neck of the magnet dewar; the step- 
per motor offers $0.1^{\circ}$ angle resolution. The source and detector are bolted rigidly to the microwave probe; subsequent connection to and from the MVNA is achieved via flexible coaxial cables [7]. In this mode of operation, one can maintain optimal coupling between the spectrometer and the cavity containing the sample, whilst rotating the probe. As discussed in great detail in ref. 7], good coupling between the various microwave elements is essential in order to maintain a high sensitivity and a low noise level. All data presented in this paper were obtained whilst sweeping the applied magnetic field at different fixed angles $\theta$ relative to the least conducting $a^{*}$ direction $(\perp b c-$ plane $)$.

\section{Results and discussion}

Figure 1 plots microwave dissipation versus magnetic field, at various angles $\theta$; the temperature was $4.3 \mathrm{~K}$ and the frequency $71.36 \mathrm{GHz}$ for these measurements. The main broad peak seen at all angles corresponds to the JPR which we have reported previously [4, [5], i.e. this mode displays all of the previously published behavior at $\theta=0^{\circ}$. The JPR peak position $\left(B_{\text {res }}\right)$ follows an approximately $1 / \cos \theta$ dependence up to $\theta \approx 80^{\circ}$, whereupon it reaches a maximum, tending towards zero-field as $\theta \rightarrow 90^{\circ}$ (see dashed curve). This re-entrant behavior of the JPR may be attributed to the in-plane field component $\left(B_{x}\right)$, as discussed in the introduction. In this same narrow angle range $\left(\theta>80^{\circ}\right)$, a new low-field resonance (LFR) develops (dotted curve), which moves to higher fields as $\theta \rightarrow 90^{\circ}$. The LFR exhibits a dramatic hysteretic behavior wherein it is only observed for up-sweeps of the magnetic field. This is illustrated in Fig. 2, where we plot the temperature dependence (raw data) of the JPR and LFR for both up- and down-sweeps of the magnetic field, with $\theta$ close to $90^{\circ}\left( \pm 0.1^{\circ}\right)$. The data in Fig. 2 data were obtained during a separate run to the data in Fig. 1, i.e. the sample is the same, but was subjected to thermal cycling to room temperature between experiments. Furthermore, the frequency of $74.20 \mathrm{GHz}$ is slightly different. Nevertheless, angle dependent studies revealed an identical behavior to the data in Fig. 1 (see Fig. 3).

We first discuss the re-entrant behavior of the JPR at angles close to $\theta=90^{\circ}$. An analytic expression for the angle dependence within the vortex liquid phase may be obtained by assuming a Gaussian form for $S(\mathbf{r})$, giving

$$
\omega_{p}^{2} \approx \omega_{p}^{2}\left(B_{z}, T\right) \exp \left(-\frac{\pi s^{2} B_{x}^{2}}{\Phi_{o} B_{z}}\right) \propto \frac{1}{B \cos \theta} \exp \left(-\frac{\pi s^{2} B \sin ^{2} \theta}{\Phi_{o} \cos \theta}\right)
$$


where $s$ is the interlayer spacing, and $\Phi_{o}$ is the flux quantum [6]. The dashed curve in Fig. 3 represents a fit to the above expression for the data obtained from the first experiment (open squares). While such a fit is reasonable, a better fit (solid curve in Fig. 3) involves a slight modification to the denominator of the pre-exponential factor in Eq. (2), such that $B \cos \theta \rightarrow B[\cos \theta+\alpha]$. The fit assumes fixed $\omega_{p}=2 \pi f$ and $B=B_{\text {res }}$, and yields $\alpha=0.1$ and a reasonable value for $s \sim 8 \stackrel{\circ}{A}$. The modification to Eq. (2), which produces a rounding of the $1 / \cos \theta$ behavior close to $\theta=90^{\circ}$, may have several explanations: i) it may reflect a limitation in the Gaussian approximation for $S(\mathbf{r})[6]$; or ii) it may indicate that the pancake vortex contribution to $\omega_{p}$ is not determined solely by $B_{z}$. The latter may suggest that a highly anisotropic 3D picture (as opposed to strictly 2D) offers the more realistic description of the superconductivity in the title compound, or this could also be indicative of paramagnetic pair breaking for large in-plane fields. Both of these effects have been considered in the context of other angle dependent studies of the superconductivity in $\kappa$ - $(\mathrm{ET})_{2} \mathrm{Cu}(\mathrm{NCS})_{2}$, such as $H_{c 2}$ 9]. The obtained value for $s$ is somewhat smaller than the actual interlayer spacing. However, the exponent in Eq. (1) is approximate, i.e. it assumes that the phase correlation length is approximately equal to the intra-layer intervortex separation, through $B_{z}$.

Next we turn to the hysteretic behavior, and the temperature dependence of the JPR and LFR seen in Fig. 2. The hysteresis is clearly a manifestation of the irreversible region of the vortex phase diagram, which extends beyond the maximum field sweep range at the lowest temperature of $3 \mathrm{~K}$; in fact, the JPR is barely seen for the up-sweep at this temperature. Eq. (2) was derived for a vortex liquid. Therefore, the re-entrance of the JPR at angles close to $90^{\circ}$ drives it into the irreversible region of the phase diagram at low temperatures (see Fig. 4 and ref. [10]); hence, its disappearance. Nevertheless, for all other temperatures and angles, the data presented here for the JPR were confined to the reversible liquid-like phase [5]. On the other hand, the LFR resides entirely within the irreversibility line [10]. We note that, although the JPR is not very pronounced at angles close to $90^{\circ}$ (Fig. 2), we do always see two distinct resonances on the up-sweeps (this is very clear in Fig. 1).

We now speculate that the LFR also corresponds to a JPR which is associated with the non-equilibrium critical state; the origin of its opposing angle dependence is not presently known. Indeed, the tendency to move to higher fields implies increasing interlayer phase 
coherence as $\theta \rightarrow 90^{\circ}$ (see discussion in ref. [5]), which is quite contrary to the expectations of Eq. (2). Looking at the temperature dependence of the resonance positions for angles close to $\theta=90^{\circ}$ (Fig. 4), one finds the same opposing trends for the two resonances, i.e. the JPR moves to higher fields with increasing temperature, indicating increased interlayer phase coherence, while the LFR exhibits the opposite behavior. Thus, within the irreversible region of the phase diagram, and for large in-plane fields $\left(\theta\right.$ close to $\left.90^{\circ}\right)$, the interlayer phase coherence decreases with increasing temperature, i.e. $\partial\left\langle\cos \varphi_{n, n+1}(\mathbf{r})\right\rangle / \partial \mathrm{T}<0$; the opposite holds for the reversible phase $\left(\partial\left\langle\cos \varphi_{n, n+1}(\mathbf{r})\right\rangle / \partial \mathrm{T}>0\right)$. These trends are entirely contrary to what we have previously observed with the applied field perpendicular to the layers, $\left(\theta=0^{\circ}\right)$ [5]. This could be related to the anisotropy of the superconductivity, i.e. the phenomenological constant $\alpha$ in our fit. However, further experimental and theoretical studies are clearly needed in order to understand this behavior, particularly in the irreversible phase.

\section{Summary and conclusions}

Within the vortex liquid phase, angle dependent electrodynamic investigations of $\kappa$ $(\mathrm{ET})_{2} \mathrm{Cu}(\mathrm{NCS})_{2}$ reveal a re-entrance of the JPR frequency, $\omega_{p}$, for fields close to alignment with the highly conducting layers. We are able to fit the angle dependence to a single analytic expression which is related to the superconducting phase correlation function. A sharp, low field, resonance is also observed in the vicinity of $\theta=90^{\circ}$, which appears to be associated with the non-equilibrium critical state.

\section{Acknowledgements}

This work was supported by NSF (DMR0196430, DMR0239481 and DMR0196461). SH is a Cottrell scholar of the Research Corporation.

* corresponding author, Email hill@phys.ufl.edu

[1] T. Ishiguro, K. Yamaji, and G. Saito, Organic Superconductors, Vol. 88 of Springer Series in Solid State Sciences (Springer-Verlag, Berlin, 1998).

[2] A. Carrington, I. J. Bonalde, R. Prozorov, R. W. Giannetta, A. M. Kini, J. Schlueter, H. H. Wang, U. Geiser, and J. M. Williams, Phys. Rev. Lett. 83, 4172 (1999), and references therein. 
[3] Y. Matsuda, M. B. Gaifullin, K. Kumagai, M. Kosugi, and K. Hirata, Phys. Rev. Lett. 78, 1972 (1997).

[4] M. Mola, J. T. King, C. P. McRaven, S. Hill, J. S. Qualls, and J. S. Brooks, Phys. Rev. B 62 , 5965 (2000).

[5] S. Hill, M. M. Mola, and J. S. Qualls, J. Phys. Condens. Matter 14, 6701 (2002).

[6] A. E. Koshelev, L. N. Bulaevskii, and M. P. Maley, Phys. Rev. B 62, 14403 (2000).

[7] M. Mola, S. Hill, P. Goy, and M. Gross, Rev. Sci. Inst. 71, 186 (2000).

[8] S. Hill, Phys. Rev. B 62, 8699 (2000).

[9] F. Zuo, J. S. Brooks, R. H. McKenzie, J. A. Schlueter, and J. M. Williams, Phys. Rev. B 61, $750(2000)$.

[10] M. Lang, F. Steglich, N. Toyota, and T. Sasaki, Phys. Rev. B 49, 15227 (1994). 


\section{Figure captions}

FIG. 1. Angle dependence of the microwave dissipation due to interlayer currents; the field orientations are indicated in the figure. Only up-sweeps are shown (see also Fig. 2). The temperature is $4.3 \mathrm{~K}$ and the frequency is $71.36 \mathrm{GHz}$.

FIG. 2. Temperature dependence (raw data) of the JPR and LFR for both up- and downsweeps of the magnetic field, with $\theta$ close to $90^{\circ}\left( \pm 0.1^{\circ}\right)$. The frequency is $74.20 \mathrm{GHz}$, and the temperatures are indicated in the figure.

FIG. 3. A plot of the JPR and LFR positions (in field) versus field orientation $(\theta)$. JPR data are included for two experimental runs, the first at a frequency of $71.36 \mathrm{GHz}$, the second at a frequency of $74.20 \mathrm{GHz}$; the temperature was $4.3 \mathrm{~K}$ for the first run, and $3.5 \mathrm{~K}$ for the second run. The dashed curve is a fit to Eq. (2) for the JPR data obtained in the first run; the solid curve is a modified fit (see text).

FIG. 4. A plot of the temperature dependence of the JPR and LFR resonance positions (in field), with $\theta$ close to $90^{\circ}\left( \pm 0.1^{\circ}\right)$. The frequency is $74.20 \mathrm{GHz}$. The dashed curve represents the irreversibility line estimated for this field orientation on the basis of published data (see open squares, and ref. [10]). 


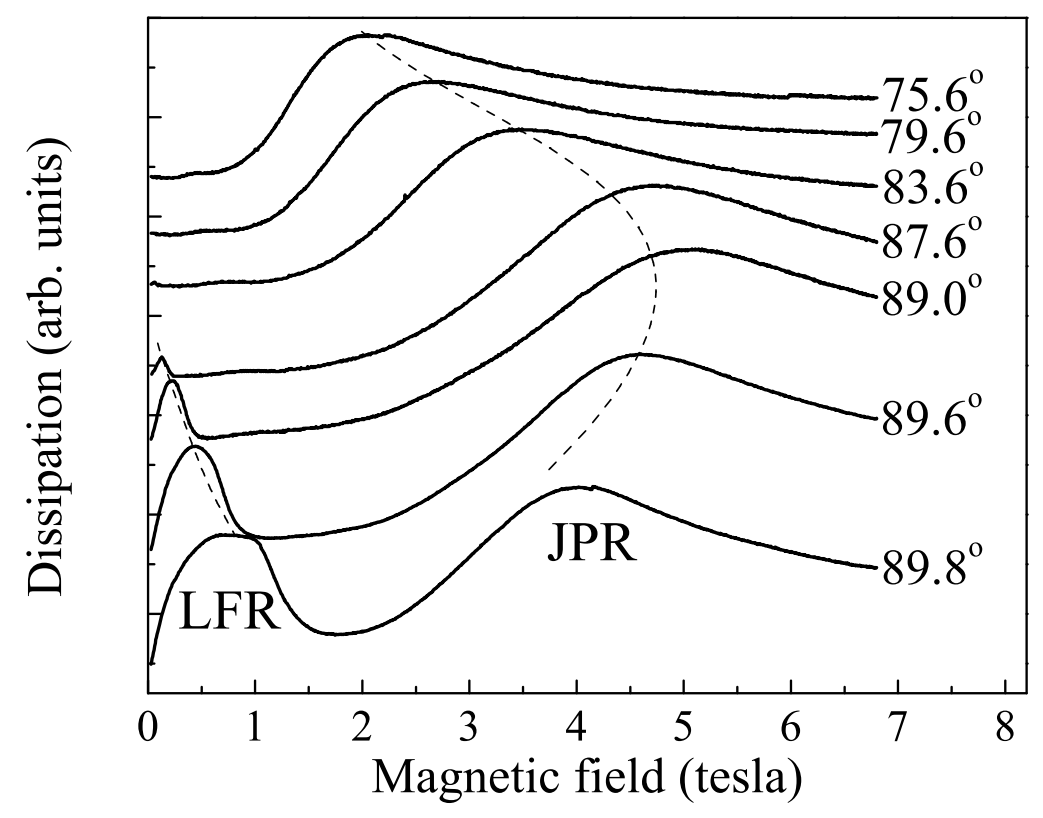

FIG. 1: Kovalev et al.

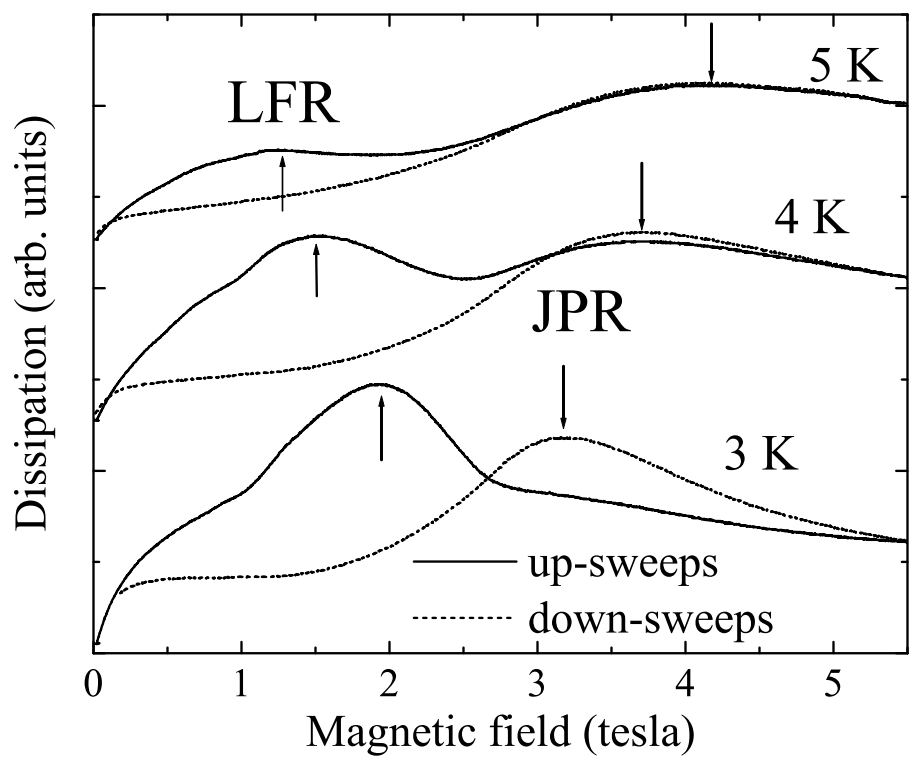

FIG. 2: Kovalev et al. 


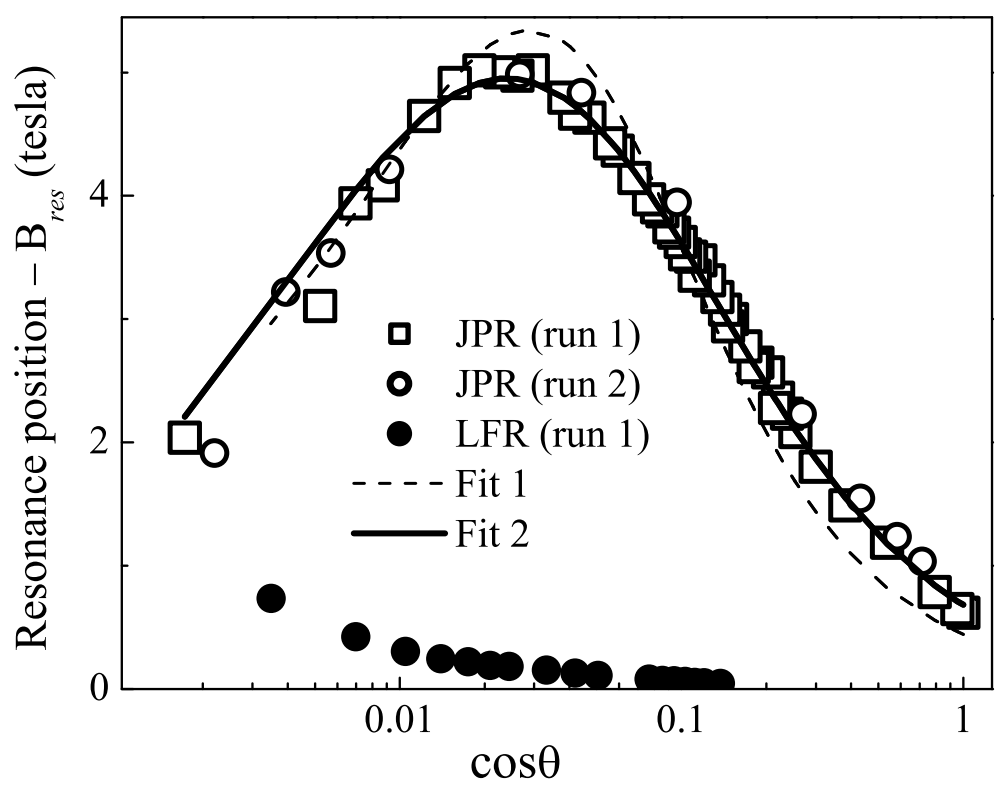

FIG. 3: Kovalev et al.

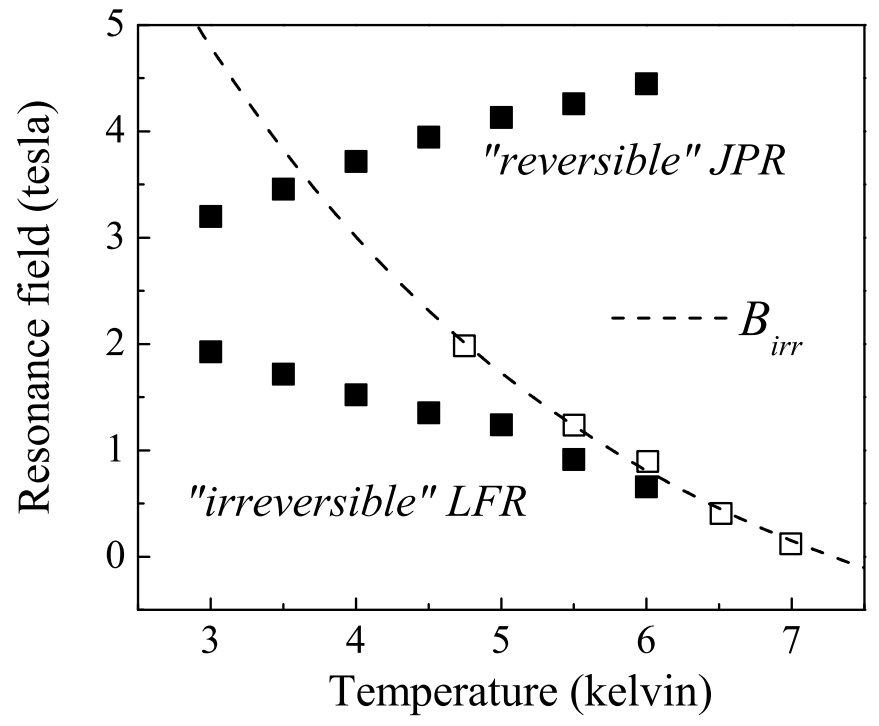

FIG. 4: Kovalev et al. 